EUROPEAN LABORATORY FOR PARTICLE PHYSICS

CERN-EP / 98-190

December 11, 1998

\title{
$\mathbf{J} / \psi, \psi^{\prime}$ and Drell-Yan production in $\mathrm{S}-\mathrm{U}$ interactions at $200 \mathrm{GeV}$ per nucleon
}

M.C. Abreu ${ }^{1, a)}$, J. Astruc ${ }^{2)}$, C. Baglin ${ }^{3)}$, A. Baldit ${ }^{4)}$, M. Bedjidian ${ }^{5)}$, P. Bordalo ${ }^{1, b)}$, A. Bohrani ${ }^{6)}$, A. Bussière ${ }^{3)}$, P. Busson ${ }^{6)}$, J. Castor ${ }^{4)}$, T. Chambon ${ }^{4)}$, C. Charlot $^{6)}$, B. Chaurand ${ }^{6)}$, I. Chevrot ${ }^{4)}$, D. Contardo ${ }^{5)}$, E. Descroix ${ }^{5, c)}$, A. Devaux ${ }^{4}$, O. Drapier ${ }^{5)}$, B. Espagnon ${ }^{4)}$, J. Fargeix ${ }^{4)}$, R. Ferreira ${ }^{1)}$, F. Fleuret ${ }^{6)}$, P. Force ${ }^{4)}$, J. Gago ${ }^{1, b)}$, C. Gerschel ${ }^{2)}$, P. Gorodetzky ${ }^{7, d)}$, J.Y. Grossiord ${ }^{5)}$, A. Guichard ${ }^{5)}$, J.P. Guillaud ${ }^{3)}$, R. Haroutunian ${ }^{5)}$, D. Jouan ${ }^{2)}$, L. Kluberg ${ }^{6)}$, R. Kossakowski' ${ }^{3)}$, G. Landaud ${ }^{4)}$, C. Lourenço ${ }^{1,8)}$, L. Luquin ${ }^{4)}$, R. Mandry ${ }^{5)}$, S. Mourgues ${ }^{4)}$, F. Ohlsson-Malek ${ }^{5, \mathrm{e})}$, S. Papillon ${ }^{2)}$, J.R. Pizzi ${ }^{5)}$, C. Racca ${ }^{7)}$, S. Ramos ${ }^{1, \mathrm{~b})}$, A. Romana ${ }^{6)}$, B. Ronceux ${ }^{3)}$, P. Saturnini ${ }^{4)}$, S. Silva ${ }^{1)}$, P. Sonderegger ${ }^{8, b)}$, X. Tarrago ${ }^{2)}$, J. Varela ${ }^{1, b, f)}$

\section{NA38 Collaboration}

\begin{abstract}
A detailed study of $\mathrm{J} / \psi, \psi^{\prime}$ and Drell-Yan production in S-U collisions has been performed by experiment NA38 at the CERN SPS. This paper presents production cross sections and their centrality dependence, based on the largest sample of S-U events collected by the experiment.
\end{abstract}


1) LIP, Av. Elias Garcia 14, P-1000 Lisbon, Portugal

2) IPN, IN2P3-CNRS and Université de Paris-Sud, F-91406 Orsay Cedex, France

3) LAPP, IN2P3-CNRS, F-74941 Annecy-le-Vieux Cedex, France

4) LPC Clermont-Ferrand, IN2P3-CNRS and Université Blaise Pascal, F-63177 Aubière Cedex, France

5) IPN Lyon, IN2P3-CNRS and Université Claude Bernard, F-69622 Villeurbanne Cedex, France

6) LPNHE, Ecole Polytechnique, IN2P3-CNRS, F-91128 Palaiseau Cedex, France

7) IRes, IN2P3-CNRS and Université Louis Pasteur, F-67037 Strasbourg Cedex, France

8) CERN, CH-1211 Geneva 23, Switzerland

a) Also at UCEH, Universidade de Algarve, Faro, Portugal

b) Also at IST, Universidade Técnica de Lisboa, Lisbon, Portugal

c) Now at Université Jean Monnet, Saint-Etienne, France

d) Now at PCC Collège de France, Paris, France

e) Now at ISN, Grenoble, France

f) Now at CERN, Geneva, Switzerland 


\section{Introduction}

Statistical QCD predicts that, at sufficiently high energy density, matter should undergo a transition from the hadronic phase to a medium of partonic degrees of freedom, where quarks and gluons are no longer confined to specific hadrons. The formation of such a medium should induce considerable changes in the normal pattern of heavy quarkonia production. In particular, the production of $\mathrm{J} / \psi$ mesons should be suppressed, either due to the colour screening of the $c \bar{c}$ potential $(\psi$ melting by 'Debye screening') or due to the breaking of the $c \bar{c}$ bound state by scattering with deconfined energetic gluons. Recent descriptions of these scenarios can be found in Ref. [1].

$\mathrm{J} / \psi$ production in high energy nucleus-nucleus interactions has been studied by experiment NA38, at the CERN SPS, since 1986. The first results obtained with $200 \mathrm{GeV}$ per nucleon oxygen and sulphur beams revealed $[2,3]$ that $\mathrm{J} / \psi$ production is indeed suppressed in ion collisions, both relative to the $\mathrm{p}-\mathrm{A}$ case and as a function of the transverse energy, $E_{\mathrm{T}}$, released in the ion collisions. However, it was later found that the suppression pattern observed in S-U collisions was compatible with a natural extrapolation of the p-A trend [4], where QGP formation is certainly not expected. To fully clarify this issue, NA38 collected a significantly larger sample of sulphur-uranium events. The corresponding results, reported in this paper, provide a very important element in the study of charmonium production and suppression, together with the results obtained in $\mathrm{p}-\mathrm{A}[5]$ and in $\mathrm{Pb}-\mathrm{Pb}[6]$ interactions.

\section{Apparatus and data reduction}

The sulphur data reported here were collected at the CERN-SPS in 1990, 1991 and 1992. The main features of the detector are recalled hereafter. Special emphasis is put on some differences with respect to the setup used in 1987, described in detail in Ref. [2].

The main component of the NA38 detector was a muon spectrometer composed of a toroidal magnet between 2 sets of multiwire proportional chambers and 4 trigger hodoscopes. In the geometrical configuration used to collect the S-U data, muon pairs were accepted in the dimuon (lab) rapidity range 2.8-4.0. The muon spectrometer was protected from the high multiplicity environment of the target region by a $520 \mathrm{~cm}$ long hadron absorber. It was made of $40 \mathrm{~cm}$ of aluminum oxyde $\left(\mathrm{Al}_{2} \mathrm{O}_{3}\right)$ followed by $480 \mathrm{~cm}$ of carbon (graphite disks) in 'setup 1', while in 'setup 2' the last $80 \mathrm{~cm}$ of carbon were replaced by iron. Besides absorbing all the hadrons produced in the interaction (including a large fraction of pions and kaons before their decay into muons), the heavier absorber imposes a higher threshold on the energy that the muons must have to trigger the experiment. In setup 2, therefore, the low mass muon pairs are strongly reduced at the trigger level, allowing to increase the intensity of the beam without affecting the aquisition dead-time. This effectively increases the statistics in the $\mathrm{J} / \psi$ mass region (see Table 1 ), degrading very little the mass resolution at the 
Table 1: Data samples collected with the two setups.

\begin{tabular}{lcccc}
\hline & $\begin{array}{c}\text { Beam intensity } \\
\text { (ions per burst) }\end{array}$ & $\begin{array}{c}\text { Interactions } \\
\text { per burst }\end{array}$ & $\begin{array}{c}\text { Recorded } \\
\text { events }\end{array}$ & $\begin{array}{c}\text { Selected } \\
\text { events }\end{array}$ \\
\hline Setup 1 & $\sim 9 \times 10^{6}$ & $\sim 1.4 \times 10^{6}$ & $9.5 \times 10^{6}$ & $2.1 \times 10^{6}$ \\
Setup 2 & $\sim 8 \times 10^{7}$ & $\sim 1.5 \times 10^{7}$ & $1.5 \times 10^{7}$ & $2.5 \times 10^{6}$ \\
\hline
\end{tabular}

$\mathrm{J} / \psi$ peak, from $\sigma=142 \mathrm{MeV}$ to $147 \mathrm{MeV}$, with an operating current of $4000 \mathrm{~A}$ in the spectrometer magnet.

In both setups the non-interacting beam was stopped by a $400 \mathrm{~cm}$ long tungsten and uranium plug located $165 \mathrm{~cm}$ downstream from the centre of the target.

The active target system in setup 1 (setup 2) consisted of 10 (12) uranium subtargets, each of them $1 \mathrm{~mm}$ thick (i.e. $1.7 \%$ of a $\mathrm{S}-\mathrm{U}$ interaction length). The targets were surrounded by 32 ring scintillators, used to identify the sub-target where the interaction occurred and to tag, for offline rejection, events where a spectator fragment has interacted in a subsequent sub-target. The small transverse dimensions of the targets $\left(1 \times 2 \mathrm{~mm}^{2}\right)$ and their large relative spacing $(24 \mathrm{~mm})$ were chosen to minimize the interaction of secondary particles (and the showering of photons and electrons) in the downstream sub-targets.

The first sub-target was larger than the others $\left(10 \times 10 \mathrm{~mm}^{2}\right)$ to intercept $100 \%$ of the incident beam, allowing to determine the targetting efficiency of the whole system. The sulphur beam had a spot size of $\sigma_{x} \simeq 300 \mu \mathrm{m}$ and $\sigma_{y} \simeq 600 \mu \mathrm{m}$. To help centering the beam on the sub-targets, the target system was aligned between two small quartz detectors, each $3 \mathrm{~mm}$ thick and subdivided into four quadrants.

A 15 radiation length electromagnetic calorimeter provided an estimate of the centrality of the collision from the measurement of the neutral transverse energy. It was made of $1 \mathrm{~mm}$ diameter scintillating plastic fibers embedded in a $14 \mathrm{~cm}$ thick lead converter in a 1:2 volume ratio. It measured the transverse energy $E_{\mathrm{T}}$ in the (lab) pseudo-rapidity range 1.7-4.1. The neutral transverse energy was obtained from the measured value after correcting for the contribution from charged hadrons. This charged component amounted to $\sim 40 \%$ according to a Monte-Carlo simulation which used a ratio 2:1 of hadronic to electromagnetic energy flux [7].

A beam hodoscope $(\mathrm{BH})$ was used to count and identify the beam particles. It was made of two consecutive planes of plastic scintillators, one divided in 16 and the other in 14 slabs. It was located $33 \mathrm{~m}$ upstream of the target, where the beam spot was large enough $\left(\sigma_{x} \simeq 3 \mathrm{~mm}, \sigma_{y} \simeq 8 \mathrm{~mm}\right)$ to intercept most of the slabs and induce acceptable individual counting rates. The average beam intensity and the number of interactions in the $4.5 \mathrm{~s}$ spill is shown in Table 1 for the two data sets. At high luminosities, the probability of having two interactions in the $20 \mathrm{~ns}$ wide reading gate of the detectors is not negligible. If not rejected, these double interactions would spoil the transverse energy measurement. Events are tagged as 'beam pile-up' when more than one incident ion crosses the $\mathrm{BH}$ in the 20 ns gate [2], but since the target thickness was $\sim 20 \% \lambda_{I}$, there is only a small fraction of this event sample where 
two interactions actually occurred. The 'interaction pile-up' sub-sample is identified through the shape analysis of the electromagnetic calorimeter signals.

The final analysis event sample is selected according to the criteria described in Refs. [2, 3, 8] which, briefly summarized, require two reconstructed tracks with a common origin in the target region, no reinteraction of a spectator fragment and no pile-up. Table 1 reports the number of events before and after applying the selection cuts.

Besides prompt dimuons (vector meson decays and $q \bar{q}$ annihilation), the measured opposite-sign muon pair event sample also includes muon pairs originating from meson decays. The correlated muon pairs from the simultaneous semileptonic decays of $D$ and $\bar{D}$ mesons must be purely estimated by Monte-Carlo simulation. On the contrary, the uncorrelated muon pairs resulting from decays of pions and kaons also contribute to the sample of like-sign muon pairs. This source of background can, therefore, be estimated from the collected data using the relation

$$
N_{\mathrm{bg}}=2 \times R_{\mathrm{bg}} \times \sqrt{N^{++} \times N^{--}},
$$

where $N^{++}\left(N^{--}\right)$is the number of positive (negative) muon pairs. Since this relation is only valid if the muon acceptance in the apparatus is independent of its charge, a fiducial cut is applied which rejects events where at least one muon, if oppositely charged, would not have been accepted. $R_{\mathrm{bg}}$ reflects eventual charge correlations among the numbers of positive and negative muons. For high multiplicity events, like those produced in $\mathrm{S}-\mathrm{U}$ collisions, $R_{\mathrm{bg}}$ is expected to be unity. The prompt dimuon signal is obtained from $N_{\text {signal }}=N^{+-}-N_{\text {bg }}$, where $N^{+-}$is the number of collected events with opposite-sign muon pairs.

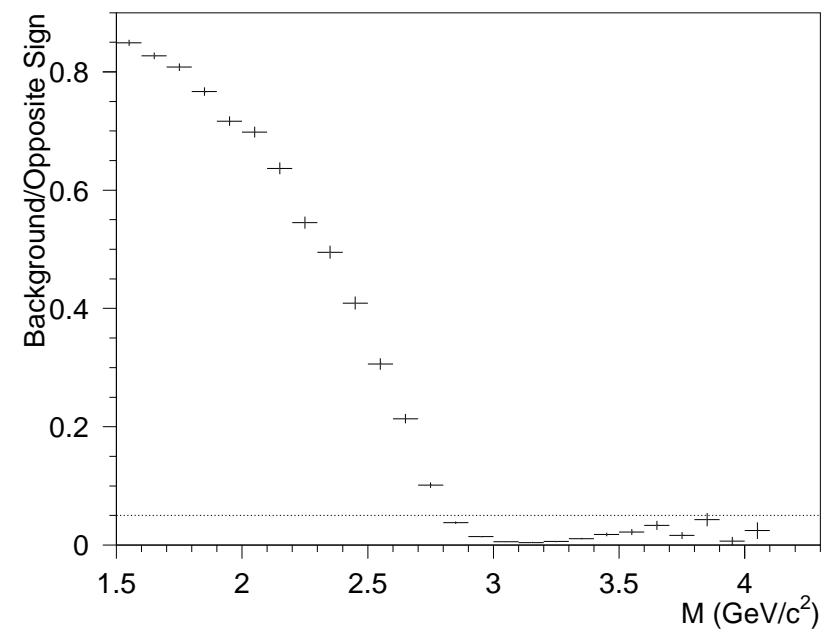

Figure 1: Background fraction in the opposite-sign muon pair sample, as a function of the mass.

Figure 1 shows that the number of background events, $N_{\mathrm{bg}}$, is a very small fraction of the number of collected opposite-sign events, $N^{+-}$, in the mass window of relevance for this paper: less than $1 \%$ in the $\mathrm{J} / \psi$ peak and less than $3 \%$ in the $\psi^{\prime}$ mass region. 


\section{$3 \quad$ Data analysis}

The aim of this study is to obtain the yield of $\mathrm{J} / \psi$ and $\psi^{\prime}$ mesons through their dimuon decay and to compare with the corresponding yield of Drell-Yan pairs. The analysis is restricted to the kinematical domain where the acceptance of the spectrometer is above $10 \%$ of its maximum value, i.e. a dimuon lab rapidity between 3 and 4 , and a dimuon polar decay angle in the Collins-Soper reference frame limited to $\left|\cos \left(\theta_{\mathrm{CS}}\right)\right|<0.5$. Figure 2 shows the muon pair invariant mass spectrum, as collected with setup 2, after background subtraction.

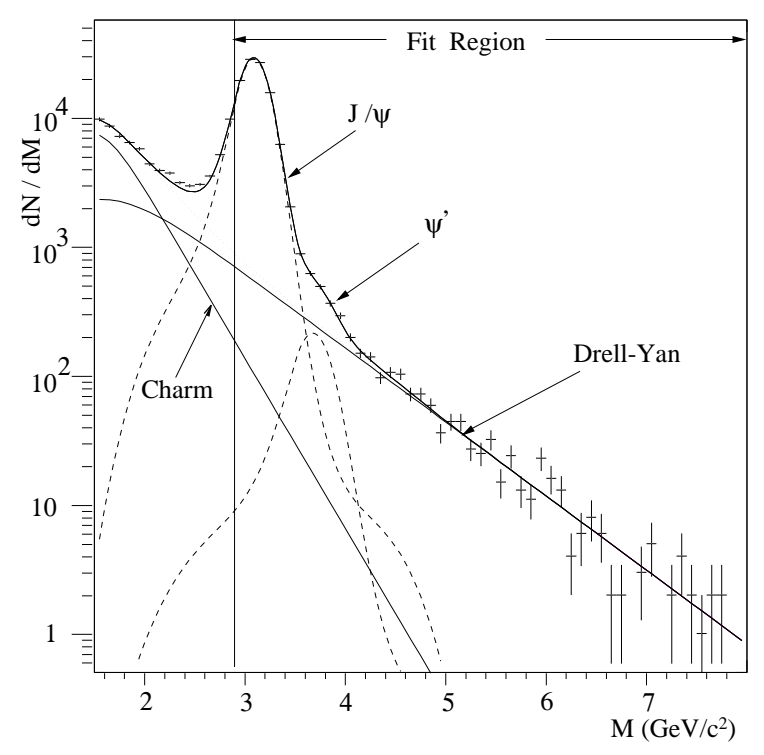

Figure 2: Signal dimuon mass spectrum. The analysis reported in this paper is limited to the window above $2.9 \mathrm{GeV} / c^{2}$.

The numbers of $\mathrm{J} / \psi, \psi^{\prime}$ and Drell-Yan events are extracted by fitting the signal dimuon mass distribution to a superposition of line shapes representing these three contributions. The line shapes are obtained by a Monte-Carlo procedure that generates events and simulates the acceptance and smearing effects, as induced by the detector. These simulated events are treated in the same way as the real events in what concerns reconstruction and selection criteria.

The charmonia resonances are generated with gaussian rapidity distributions (of width 0.6$)$ and with transverse momenta given by $m_{\mathrm{T}} \cdot K_{1}\left(\frac{m_{\mathrm{T}}}{\mathrm{T}}\right)($ with $\mathrm{T}=236 \mathrm{MeV})$. The quoted parameter values were found through an iteration procedure that resulted in a good agreement between the resulting rapidity and $p_{\mathrm{T}}$ shapes and the measured $\mathrm{J} / \psi$ distributions [9]. The charmonia events are generated according to a uniform $\cos \left(\theta_{\mathrm{CS}}\right)$ distribution.

The (correlated) mass and rapidity distributions of the Drell-Yan events are generated with the LO expression, using the GRV LO 92 set of parton distribution functions [10]. The polar decay angle follows the usual $1+\cos ^{2}\left(\theta_{\mathrm{CS}}\right)$ distribution 
while the transverse momentum is generated with the same function as the $\mathrm{J} / \psi$, but with a variable dimuon mass.

It is important to note that the results presented in this paper are completely insensitive to the exact shape of the $p_{\mathrm{T}}$ distributions used to generate the events. Indeed, the acceptance of the muon spectrometer is rather flat as a function of $p_{\mathrm{T}}$, down to $p_{\mathrm{T}}=0$ for dimuon masses above $\sim 2 \mathrm{GeV} / c^{2}$, making the integrated acceptances independent of the generation function.

The intermediate mass continuum (between the $\phi$ and the $\mathrm{J} / \psi$ peaks) produced in $\mathrm{p}-\mathrm{A}$ and $\mathrm{S}-\mathrm{U}$ interactions has been analysed as a superposition of muon pairs originating from the Drell-Yan mechanism and from semi-leptonic decays of $D$ and $\bar{D}$ mesons $[9,11]$. From those studies we know that the mass spectra can be very well described by the superposition of these expected sources, if the normalisation of the $D \bar{D}$ contribution is fitted from the data.

In order to keep the charmonia analysis as insensitive as possible to the physical understanding of the lower mass continuum, the dimuon mass distributions are fitted in the window above $2.9 \mathrm{GeV} / c^{2}$. The normalisation of the $D \bar{D}$ curve is previously fixed from a fit to the mass window $1.5-2.0 \mathrm{GeV} / c^{2}$ and, as can be appreciated from Fig. 2, has a negligible effect in the analysis of the high mass region.

The analytical curves presented in Fig. 2 are empirical parametrisations of the mass distributions of the four simulated physical processes, as they appear after the acceptance and smearing effects induced by the spectrometer [12].

In view of the extremely high statistics collected in the $\mathrm{J} / \psi$ peak, the Monte-Carlo simulation shapes must be slightly adjusted to precisely reproduce the measured $\mathrm{J} / \psi$ shape, to prevent any bias on the results of the fit in terms of yields. This is done by leaving as free parameters in the fit the mean, $\mu$, and width, $\sigma$, of the $\mathrm{J} / \psi$ "gaussian" [12]. These two parameters are also used to adjust the analytical function that describes the $\psi^{\prime}$ simulated shape, imposing $\mu^{\prime}=\mu+0.589 \mathrm{GeV} / c^{2}$ and $\sigma^{\prime}=\sigma \frac{\sigma_{\text {sim }}^{\prime}}{\sigma_{\text {sim }}}$, where $\sigma_{\text {sim }}\left(\sigma_{\text {sim }}^{\prime}\right)$ is the width of the simulated $\mathrm{J} / \psi\left(\psi^{\prime}\right)$ peak.

A maximum likelihood fit to the dimuon mass distribution provides the values of $\mu$ and $\sigma$, the number of $\mathrm{J} / \psi$ events, $N_{\psi}$, and the ratios $N_{\psi^{\prime}} / N_{\psi}$ and $N_{\psi} / N_{\text {DY }}$, where $N_{\psi^{\prime}}$ and $N_{\mathrm{DY}}$ are the numbers of $\psi^{\prime}$ and Drell-Yan events, respectively. The result of the best fit is included in Fig. 2.

\section{Absolute cross sections and ratios}

The absolute cross section of $\mathrm{J} / \psi$ production in $\mathrm{S}-\mathrm{U}$ collisions was determined from an event sample collected with setup 1, for which the luminosity has been precisely measured [9].

The incident flux of Sulphur ions is determined by the beam hodoscope. A small fraction of the beam flux, $3.15 \%$, interacts in the quartz beam detector, BI, located just upstream from the target. These interactions are identified by the active target system and rejected from the analysis event sample. The calculation of the integrated 
luminosity takes into account this absorption factor.

The effective length of the target is calculated from the length of the first subtarget (which intercepts all the beam profile) using the ratio between the number of events produced in all the sub-targets and in the first sub-target. This ratio is computed using only the events with a muon pair in the mass range $2.7-3.5 \mathrm{GeV} / c^{2}$, where the background contribution can be neglected.

The efficiency of the algorithm that recognises the sub-target where the interaction took place depends on the number of charged particles released in the collisions and, therefore, on $E_{\mathrm{T}}$. Very peripheral S-U collisions, with $E_{\mathrm{T}}$ below $13 \mathrm{GeV}$, have a subtarget recognition efficiency lower than $15 \%$ and were rejected. We have estimated the fraction of events surviving this cut, $92 \pm 1 \%$, using the $E_{\mathrm{T}}$ distribution before applying the sub-target recognition algorithm. We must correct for this 'acceptance' to obtain the total (all $E_{\mathrm{T}}$ ) cross sections. For the selected event sample, the average efficiency to identify the interaction sub-target is $84 \pm 3 \%$.

The fraction of events that survive the pile-up selection cut is $92 \pm 1 \%$, while for $87 \pm 1 \%$ of the events no reinteraction is detected in a downstream sub-target.

To calculate the $\mathrm{J} / \psi$ absolute cross section, we also need to take into account the track reconstruction efficiency $(95 \pm 4 \%)$, the trigger efficiency $(94 \pm 4 \%)$ and the acceptance of setup 1 for $\mathrm{J} / \psi$ production, $19.8 \%$. The total systematic error affecting the absolute cross sections amounts to $\sim 8 \%$.

From a sub-sample of events collected with the setup 1, in the kinematical domain $3.0<y<4.0$ and $\left|\cos \left(\theta_{\mathrm{CS}}\right)\right|<0.5$, we obtain $B \sigma_{\psi}=7.78 \pm 0.04 \pm 0.62 \mu \mathrm{b}$ for the $\mathrm{J} / \psi$ cross section (multiplied by the branching ratio into muons).

This measurement is in good agreement with the value previously published [13] by NA38, based on the data collected in 1987, once extrapolated to the whole $\cos \left(\theta_{\mathrm{CS}}\right)$ domain and to $x_{F}>0$.

Table 2: Acceptances and number of events of setup 2, after quality and kinematical selection cuts, for $\mathrm{J} / \psi, \psi^{\prime}$ and Drell-Yan (in the $2.9-4.5 \mathrm{GeV} / c^{2}$ mass window).

\begin{tabular}{ccr}
\hline & A (\%) & Events \\
\hline $\mathrm{J} / \psi$ & 16.0 & 113452 \\
$\psi^{\prime}$ & 18.4 & 936 \\
$\mathrm{DY}$ & 17.1 & 4808 \\
\hline
\end{tabular}

From the high statistics event sample collected with setup 2 (see Table 2), we have measured very precisely the ratios $B \sigma_{\psi^{\prime}} / B \sigma_{\psi}=0.76 \pm 0.04 \%$ and $B \sigma_{\psi} / \sigma_{\mathrm{DY}}=$ $25.1 \pm 0.8$, integrating the Drell-Yan cross section between 2.9 and $4.5 \mathrm{GeV} / c^{2}$. It is important to note that systematical errors arising from normalisation uncertainties do not affect these ratios.

From these ratios and the $\mathrm{J} / \psi$ absolute cross section, we have computed the $\psi^{\prime}$ and the Drell-Yan cross sections: $B \sigma_{\psi^{\prime}}=59.1 \pm 6.2 \pm 4.7 \mathrm{nb}$ and $\sigma_{\mathrm{DY}}=310 \pm 10 \pm 25 \mathrm{nb}$ (in the mass window $2.9-4.5 \mathrm{GeV} / c^{2}$ ). 


\section{Centrality dependence of charmonia production}

In the context of using charmonia suppression as a signature of QGP formation in nucleus-nucleus collisions, it is very important to understand how the production yields change from peripheral to central collisions. This study is considerably facilitated by using as a reference the production of (high mass) Drell-Yan dileptons, a well known process, linearly proportional to the number of nucleon-nucleon collisions and not expected to be affected by QGP formation.

We use the neutral transverse energy measured by the electromagnetic calorimeter, $E_{\mathrm{T}}$, to study charmonia production in S-U interactions as a function of the centrality of the collision. The transverse energy distribution for Drell-Yan pairs with an invariant mass above $4 \mathrm{GeV} / \mathrm{c}^{2}$ is shown in Fig. 3. The spectrum is corrected for the target identification inefficiency and is normalized to the integrated cross section value.

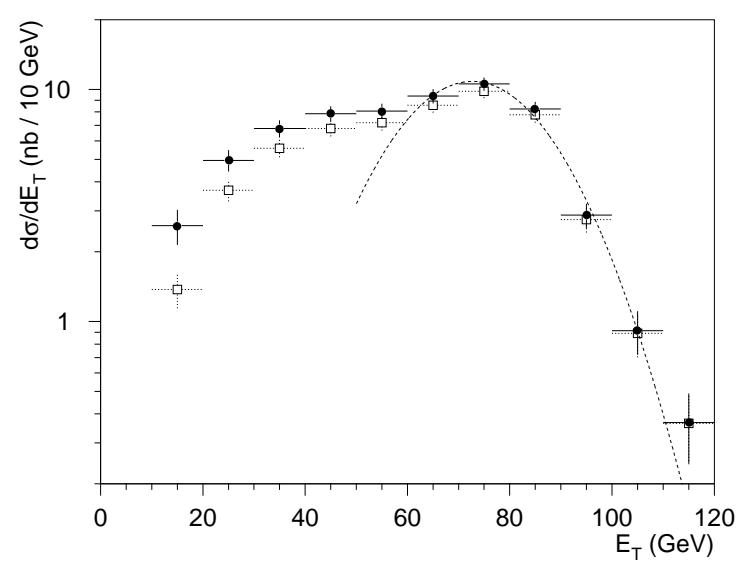

Figure 3: Transverse energy distribution for events with $M>4 \mathrm{GeV} / c^{2}$, before (open squares) and after (filled circles) sub-target inefficiency correction.

The total sample of muon pair events is divided into 5 approximately equipopulated sub-samples, according to the transverse energy of the event. Table 3 includes the $E_{\mathrm{T}}$ ranges of each bin. Events above $E_{\mathrm{T}}=88 \mathrm{GeV}$, in the tail of the $E_{\mathrm{T}}$ distribution (see Fig. 3), have not been used in this centrality dependence study.

The mass distributions obtained for each sub-sample of events are fitted as previously explained, using as free parameters the total number of $\mathrm{J} / \psi$ events and the ratios $B \sigma_{\psi} / \sigma_{\mathrm{DY}}$ and $B \sigma_{\psi^{\prime}} / B \sigma_{\psi}$. The $\mu$ and $\sigma$ parameters are fixed to the values obtained from the total sample of events. Table 3 shows the fitted values.

Figure 4 shows the $E_{\mathrm{T}}$ dependence of the ratios $B \sigma_{\psi} / \sigma_{\mathrm{DY}}$ and $B \sigma_{\psi^{\prime}} / \sigma_{\mathrm{DY}}$. Both ratios decrease significantly as $E_{\mathrm{T}}$ increases, i.e. as the interaction becomes more and more central.

The $B \sigma_{\psi^{\prime}} / B \sigma_{\psi}$ ratio has been shown to be constant from pp to $\mathrm{p}-\mathrm{U}$ [5], with an average value of $1.64 \pm 0.03 \%$. The band on the right side of Fig. 4 has been 
Table 3: Centrality dependence of $N_{\psi}$ and of the ratios $\psi / D Y$ and $\psi^{\prime} / \psi$.

\begin{tabular}{cccc}
\hline $\begin{array}{c}E_{\mathrm{T}} \\
(\mathrm{GeV})\end{array}$ & $N_{\psi}$ & $B \sigma_{\psi} / \sigma_{\mathrm{DY}}$ & $\begin{array}{c}B \sigma_{\psi^{\prime}} / B \sigma_{\psi} \\
(\%)\end{array}$ \\
\hline $13-88$ & $113288 \pm 543$ & $25.2 \pm 0.8$ & $0.76 \pm 0.08$ \\
\hline $13-34$ & $18265 \pm 154$ & $29.7 \pm 2.4$ & $1.15 \pm 0.18$ \\
$34-50$ & $20969 \pm 167$ & $26.9 \pm 1.8$ & $0.91 \pm 0.17$ \\
$50-64$ & $21240 \pm 168$ & $24.7 \pm 1.6$ & $0.77 \pm 0.15$ \\
$64-77$ & $23430 \pm 177$ & $23.7 \pm 1.4$ & $0.56 \pm 0.14$ \\
$77-88$ & $17684 \pm 154$ & $22.6 \pm 1.5$ & $0.39 \pm 0.15$ \\
\hline
\end{tabular}
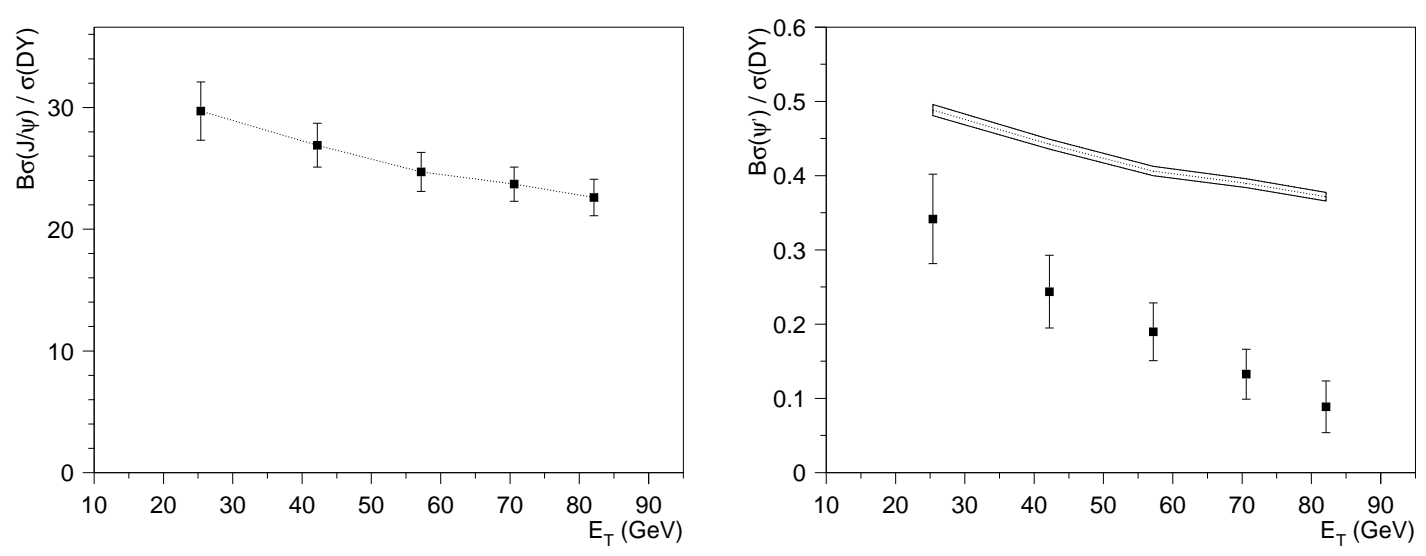

Figure 4: Ratio of $\mathrm{J} / \psi$ (left) and $\psi^{\prime}$ (right) to Drell-Yan cross sections as a function of $E_{\mathrm{T}}$. The Drell-Yan cross section is given in the mass range $2.9-4.5 \mathrm{GeV} / c^{2}$.

derived from the $B \sigma_{\psi} / \sigma_{\mathrm{DY}}$ pattern assuming that the same value would still hold in $\mathrm{S}-\mathrm{U}$ reactions. The $\psi^{\prime} / \psi$ ratio measured in $\mathrm{S}-\mathrm{U}$ collisions is more than a factor of 2 lower than the $\mathrm{p}-\mathrm{A}$ value, and the difference is seen to be more pronounced in the most central interactions. While in p-A collisions the nuclear medium has exactly the same influence on the two charmonia states, as if they were the same (preresonant) state, in the case of $\mathrm{S}-\mathrm{U}$ collisions the $\mathrm{J} / \psi$ and $\psi^{\prime}$ (fully formed) resonances clearly suffer different interactions with the surrounding matter.

It is important to compare these results on the centrality dependence of charmonia production with model calculations and with results from other experiments. However, it is not easy to directly compare the $E_{\mathrm{T}}$ distributions measured by NA38 with theoretical calculations of $E_{\mathrm{T}}$ or with other centrality related variables, measured by other experiments. This emphasizes the importance of converting the measured transverse energy in a variable more directly related to the geometry of the nucleusnucleus collisions, as the impact parameter, $b$.

The relation between the neutral transverse energy and the impact parameter of the reaction has been determined with a geometrical model that properly describes the S-U $E_{\mathrm{T}}$ spectra. This simulation is based on an improved version of the model 
described in Ref. [8]. The transverse energy is computed as being proportional to the number of wounded nucleons $[14,15]$, calculated for each impact parameter from the overlap of the nuclear density distributions. We have used the Woods-Saxon nuclear densities with the numerical values tabulated in Ref. [16]. The calculation incorporates gaussian fluctuations in the correlation between the number of wounded nucleons and the released transverse energy. The resulting correlation between $E_{\mathrm{T}}$ and the impact parameter can be seen on the left side of Fig. 5, while the average values of $b$, and of $E_{\mathrm{T}}$, are collected in Table 4, for each of the five centrality bins used in this paper.
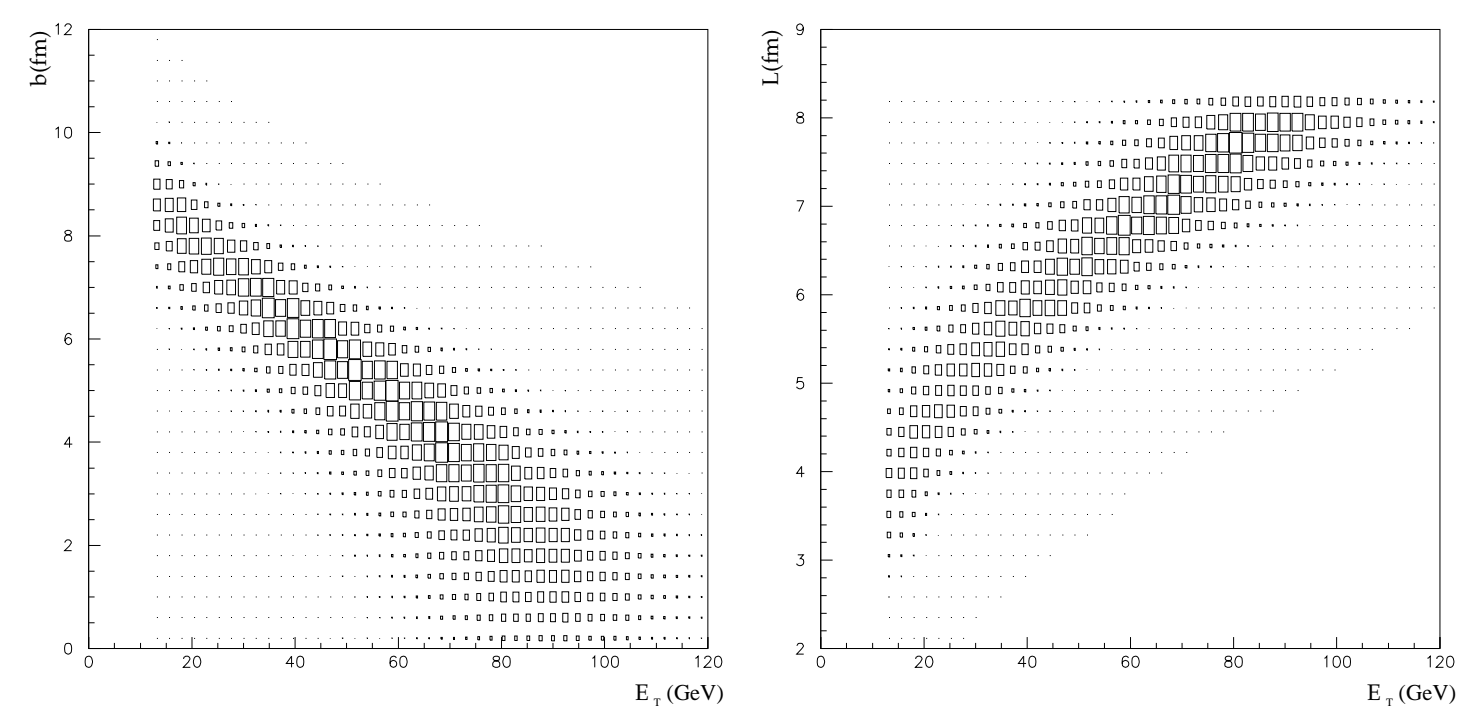

Figure 5: Correlation between $E_{\mathrm{T}}$ and the impact parameter of the reaction (left) and the length of matter crossed by the (preresonant) charmonia state (right).

Table 4: Average values of $E_{\mathrm{T}}$, impact parameter and $L$, for each $E_{\mathrm{T}}$ bin. The error bar given with $L$ is the r.m.s. value corresponding to the width of the $E_{\mathrm{T}}$ bin.

\begin{tabular}{ccc}
\hline$\left\langle E_{\mathrm{T}}\right\rangle(\mathrm{GeV})$ & $b(\mathrm{fm})$ & $L(\mathrm{fm})$ \\
\hline 25.4 & $7.5 \pm 0.7$ & $4.8 \pm 0.6$ \\
42.2 & $6.0 \pm 0.7$ & $6.0 \pm 0.4$ \\
57.2 & $4.8 \pm 0.8$ & $6.7 \pm 0.4$ \\
70.6 & $3.6 \pm 1.0$ & $7.3 \pm 0.4$ \\
82.1 & $2.6 \pm 1.0$ & $7.6 \pm 0.3$ \\
\hline
\end{tabular}

It has been empirically observed [4] that the measured $\mathrm{J} / \psi$ yields follow a simple exponential scaling as a function of the length of nuclear matter, $L$, crossed by the charmonia state. This purely geometrical variable, fully determined from the impact parameter of the collision, has been determined by a geometrical simulation similar to the one described above. The calculated correlation between $E_{\mathrm{T}}$ and $L$ is presented on the right side of Fig. 5, while the average values of $L$ in our five $E_{\mathrm{T}}$ bins are included in Table 4. 
The dependence of charmonia production on the centrality of the S-U interactions, as parametrised by the variable $L$, is shown in Fig. 6 . The scaling of the $\mathrm{J} / \psi$ yields on $L$ suggests [4] that the suppression of charmonia production relative to the Drell-Yan yield is due to final state absorption of the $c \bar{c}$ states while moving through the nuclear matter. Within this interpretation, the pattern of charmonium suppression should be, to first order, an exponentially decreasing function of $L$.
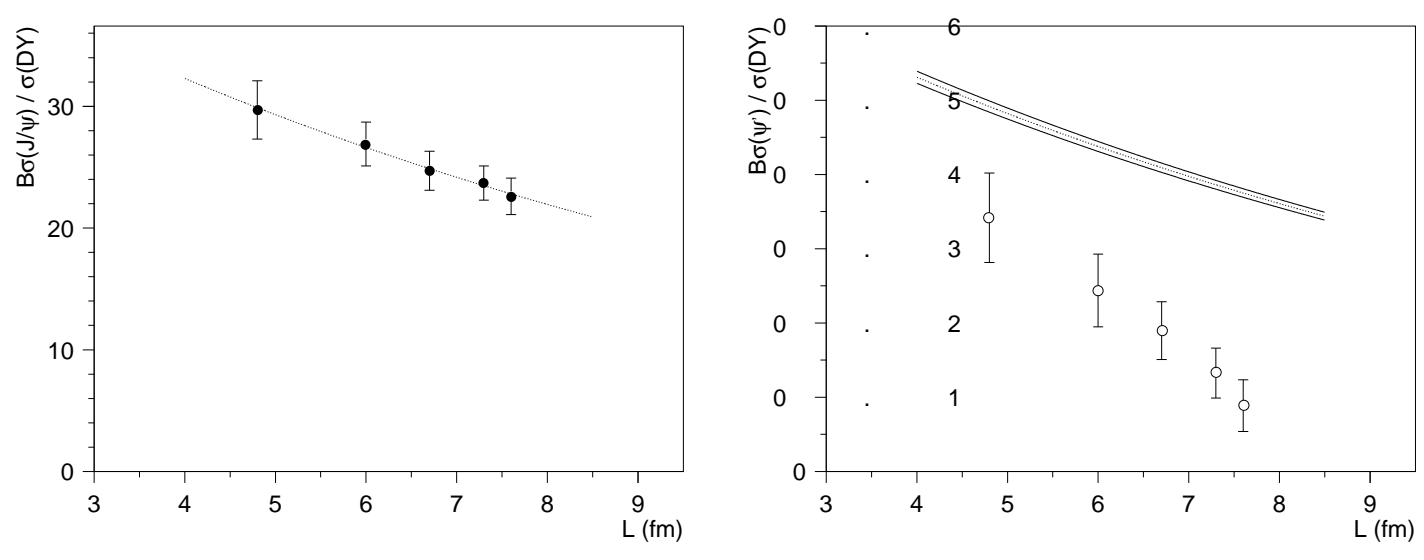

Figure 6: Dependence of the $B \sigma_{\psi} / \sigma_{\mathrm{DY}}$ and $B \sigma_{\psi^{\prime}} / \sigma_{\mathrm{DY}}$ ratios on the variable $L$. The line on the left figure is the best fit to the expression $\exp \left(-\rho \sigma_{\mathrm{abs}} L\right)$. The band in the right figure corresponds to the same line, scaled by $1.64 \pm 0.03 \%$.

Fitting the $\mathrm{J} / \psi$ data to the $\operatorname{expression} \exp \left(-\rho \sigma_{\text {abs }} L\right)$, and using an average nuclear density of $\rho=0.17$ nucleons $/ \mathrm{fm}^{3}$, we obtain an absorption cross section of $\sigma_{\mathrm{abs}}^{\psi}=5.68 \pm 1.92 \mathrm{mb}$. Only statistical errors were taken into account since this value is fully determined by the relative variation of the $\mathrm{J} / \psi$ yield from peripheral to central S-U collisions. A more refined treatment of the collision geometry (see Ref. [15]) leads to an absorption cross-section $\sim 13 \%$ higher than the value obtained in the simplified procedure described here.

If we apply the same procedure to the $\psi^{\prime}$ data we find a four times bigger absorption cross section, quantifying the stronger suppression of $\psi^{\prime}$ production in S-U collisions.

\section{Conclusions}

Charmonia and Drell-Yan production cross sections have been measured in S-U interactions, at $\sqrt{s}=20 \mathrm{GeV}$. In the kinematical domain defined by $3.0<y<4.0$ and $\left|\cos \left(\theta_{\mathrm{CS}}\right)\right|<0.5$ we obtain $B \sigma_{\psi}=7.78 \pm 0.04 \pm 0.62 \mu \mathrm{b}, B \sigma_{\psi^{\prime}} / B \sigma_{\psi}=0.76 \pm 0.04 \%$ and $B \sigma_{\psi} / \sigma_{\mathrm{DY}}=25.1 \pm 0.8$, integrating the Drell-Yan cross section between 2.9 and $4.5 \mathrm{GeV} / c^{2}$. The $\mathrm{J} / \psi$ production cross-section per nucleon-nucleon collision decreases with increasing centrality. A significantly steeper decreasing pattern is observed for the $\psi^{\prime}$ resonance. 


\section{References}

[1] H. Satz, in "International School of Subnuclear Physics", A. Zichichi (Ed.), Erice, Italy, 1997, BI-TP-97-47, hep-ph/9711289.

[2] C. Baglin et al. (NA38 Coll.), Phys. Lett. B 220 (1989) 471.

[3] C. Baglin et al. (NA38 Coll.), Phys. Lett. B 255 (1991) 459.

[4] C. Gerschel and J. Huefner, Z. Phys. C 56 (1992) 71.

[5] M.C. Abreu et al. (NA38 Coll.), accepted by Phys. Lett. B

[6] M.C. Abreu et al. (NA50 Coll.), Phys. Lett. B 410 (1997) 327;

M.C. Abreu et al. (NA50 Coll.), Phys. Lett. B 410 (1997) 337.

[7] C. Barrière, Ph.D. Thesis, Université Blaise Pascal, Clermont-Ferrand (1993);

L. Luquin, Ph.D. Thesis, Université Blaise Pascal, Clermont-Ferrand (1995).

[8] C. Baglin et al. (NA38 Coll.), Phys. Lett. B 251 (1990) 465.

[9] C. Lourenço, Ph.D. Thesis, Universidade Técnica de Lisboa, Lisbon (1995).

[10] M. Glück, E. Reya and A. Vogt, Zeit. Phys. C 53 (1992) 127;

M. Glück, E. Reya and A. Vogt, Phys. Lett. B 306 (1993) 391.

[11] M.C. Abreu et al. (NA38 Coll.), Nucl. Phys. A 566 (1994) 77c;

A. Borhani, Ph.D. Thesis, Ecole Polytechnique, Palaiseau (1996).

[12] F. Fleuret, Ph.D. Thesis, Ecole Polytechnique, Palaiseau (1997).

[13] C. Baglin et al. (NA38 Coll.), Phys. Lett. B 270 (1991) 105.

[14] A. Bialas et al., Nucl. Phys. B 111 (1976) 461.

[15] D. Kharzeev et al., Z. Phys. C 74 (1997) 307.

[16] C.W. de Jager et al., Atomic Data and Nuclear Data Tables 14 (1974) 485. 